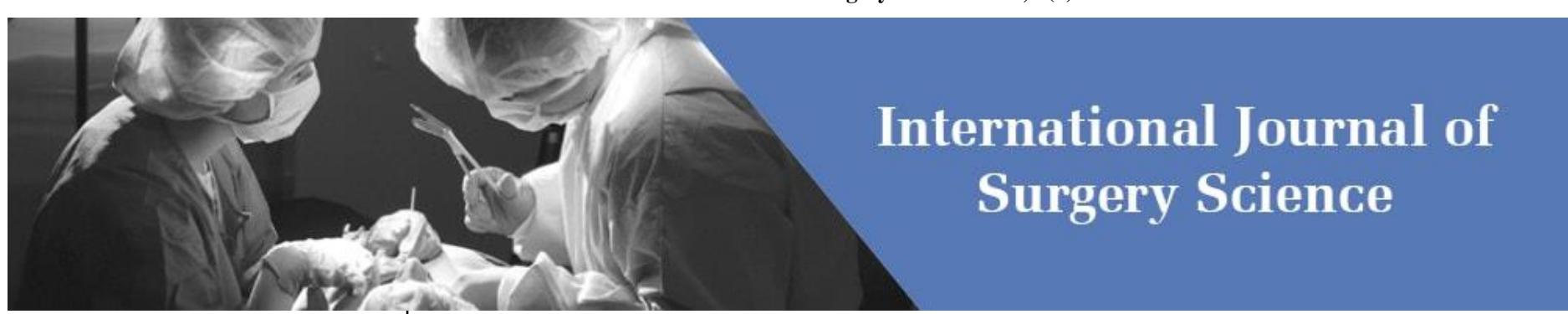

E-ISSN: 2616-3470

P-ISSN: 2616-3462

(C) Surgery Science

www.surgeryscience.com

2021; 5(4): 151-155

Received: 19-08-2021

Accepted: 21-09-2021

Dr. Kavuru Naga Siri

Department of General Medicine

Sree Balaji Medical College and

Hospital, Chennai, Tamil Nadu,

India

Dr. Bommakanti Sai Arpita M.B.B.S, Malla Reddy Institute of

Medical Sciences, Hyderabad,

Telangana, India

Dr. Muppulla NV Neelendra

Department of Surgical

Gastroenterology, Sri

Ramachandra Medical College,

Chennai, Tamil Nadu, India
Corresponding Author:

Dr. Bommakanti Sai Arpita

M.B.B.S, Malla Reddy Institute of

Medical Sciences, Hyderabad,

Telangana, India

\section{Burden of peptic ulcer disease among patients with upper gastrointestinal bleeding}

\section{Dr. Kavuru Naga Siri, Dr. Bommakanti Sai Arpita and Dr. Muppulla NV Neelendra}

DOI: https://doi.org/10.33545/surgery.2021.v5.i4c.778

\section{Abstract}

Background: Peptic ulcer disease continues to be the most common cause of non-variceal upper gastrointestinal bleeding. The clinical presentation varies depending on the intensity of bleeding. It can range from an occult bleed to melena or hematemesis and haemorrhagic shock. The diagnostic procedure Esophagogastroduodenoscopy is gold standard for confirmation of peptic ulcer disease and is also helpful in identification of bleeding source. Irrespective of recent advances in medical treatment of peptic ulcer disease, it is important to quickly identify any signs and symptoms of bleeding. This allows for appropriate management of the bleed at an early stage. Therefore, it is important to know the frequency of peptic ulcer disease amongst upper GI bleeds.

Aims and Objectives: The main aim of the current study is to identify the burden of peptic ulcer disease amongst patients with upper gastro-intestinal bleeding.

Methodology: This retrospective study on 100 selected patients was conducted at the gastrointestinal unit, department of General Medicine, in a South Asian tertiary care centre, over a period of 6 months between May 2020 to December 2020.This period includes review of literature, collection and analysis of data, discussion and finalization of the report. 100 selected patients presenting to the outpatient department with GI symptoms and upper gastrointestinal bleeding, both male and female and above 18 years of age were enrolled in the study. The patient's medical history was recorded and diagnosis of peptic ulcer disease was confirmed by Esophagogastroduodenoscopy (EGD).

Results: Out of 100 investigated cases of upper gastrointestinal bleeding, frequency of peptic ulcer disease was 53, of which 24 were duodenal ulcer, 21 were gastric ulcer, 7 were with both gastric and duodenal ulcer and 1 was an esophageal ulcer. The age distribution in the study population ranged from 30 years to 90 years. With the youngest being 33 years of age and oldest 86years. The mean age was 55 years. Out of 100 cases 76 were males and 24 were females, giving a M: F ratio of 3:1.Risk factors included alcohol intake which was seen in 32 patients, smoking was seen in 41, and NSAID's use was seen in 8 patients; out of a total of 100 patients.

Conclusion: Knowing the burden of peptic ulcer disease amongst upper gastrointestinal bleeds makes us aware of the morbidity it can cause. This makes us more alert and ensures mandatory evaluation for bleeding in any suspected or confirmed case of peptic ulcer disease.

Keywords: peptic ulcer disease, non-variceal upper gastrointestinal bleeding and esophagogastroduodenoscopy (EGD)

\section{Introduction}

An ulcer is a break in the continuity of the surface epithelium of skin or mucus membrane, caused due to molecular death. Peptic ulcers are an area of open sore usually found in the lining of stomach or intestine. In a broad sense the word "peptic" refers to an ulcer caused due to effects of stomach acid. On gross examination, a peptic ulcer is usually less than $2 \mathrm{~cm}$ in diameter, classically round to oval in shape, with a punched out and sharply defined margins. Due to peptic digestion, there is no exudate- the base of ulcer appears to be smooth with visible blood vessels. The mucosal margins may go beyond the margin of the base. Gastric mucosal folds radiate from the ulcer margin. Microscopically, the ulcer surface collects slough and inflammatory debris, below which there is intense neutrophilic infiltration along with active granulation tissue. Fibrinoid necrosis may been seen. Vessels within the ulcer are thickened and thrombosed.

The more common types of peptic ulcer are Gastric, Duodenal, and Esophageal. Widely known causes of peptic ulcer disease includes the bacteria $H$. pylori and frequent, 
unregulated use of NSAID's. Rare conditions like ZollingerEllison syndrome, antral $G$ cell hyperplasia cause ulcers in the distal small intestine, beyond the duodenum. Despite improved hygiene practices to prevent feco-oral transmission of $H$. pylori and increased awareness of misuse of NSAID's, peptic ulcer disease remains a cause for concern in many individuals.

In a systematic review of 31 published studies, the pooled incidence of uncomplicated peptic ulcer disease was approximately one case per 1000 person-years in the general population, and the incidence of ulcer complications was approximately 0.7 cases per 1000 person-years ${ }^{[1]}$. Peptic ulcer disease is a global problem with a lifetime risk of development ranging from $5 \%$ to $10 \%{ }^{[2]}$. Duodenal ulcers are four times more common than gastric ulcers ${ }^{[3]}$.

A peptic ulcer can lead to complications if left untreated or if the contributing factors are not eliminated. These include upper gastrointestinal bleeding, perforation, penetration, and malignant transformation. With the introduction of $\mathrm{H} 2$ receptor antagonists and proton pump inhibitors, the incidence of elective surgery for peptic ulcer (PU) disease has decreased dramatically, although complications of peptic ulcer disease such as perforation and bleeding have remained fairly constant ${ }^{[4]}$. A peptic ulcer can bleed chronically causing anemia due to blood loss. An acute bleed from an ulcer can present as hematemesis. A brisk bleed can present with melena or uncommonly hematochezia. Peptic ulcer disease remains one of the most common cause of upper GI bleed till date.

\section{Aims and Objectives}

1. To identify the burden of peptic ulcer disease in patients with upper gastrointestinal bleeding.

2. To analyse the distribution of type of peptic ulcer causing upper gastrointestinal bleed.

3. To describe the frequency distribution of study population based on factors like gender, age and risk factors.

\section{Materials and Methods}

A retrospective study was conducted at the gastrointestinal unit, department of General Medicine, in a South Asian tertiary care centre, over a period of 6 months between May 2020 to December 2020.This period includes review of literature, collection and analysis of data, discussion and finalization of the report. 100 selected patients presenting to the outpatient department with GI symptoms and upper gastrointestinal bleeding were enrolled in the study. History pertaining to the patient's illness, including exposure to risk factors and past medical history was obtained in the form of a questionnaire. Using excel, the questionnaire was converted into a data entry user interface. The patients were then evaluated for presence of peptic ulcer disease based on presenting symptoms: 1) epigastric pain 2) heart burn 3) regurgitation 4) pain abdomen 5) early satiety. Diagnosis of peptic ulcer was confirmed by Esophagogastroduodenoscopy (EGD) using a gastroscope of $9.8 \mathrm{~mm}$ diameter with a channel size of $2.8 \mathrm{~mm}$. Relevant clinical data was analysed and results were tabulated.

Inclusion criteria: The study was limited to those who clinically presented with upper gastrointestinal bleed (Hematemesis, Melena or both. Those with a positive Faecal occult blood test (FOBT) which was done for evaluation of anemia.) and any accompanying GI symptoms (pain abdomen, epigastric pain, heart burn, bloating, regurgitation, and early satiety).

Patients of either gender and $\geq$ age of 18 years were included in the study after taking written consent.

\section{Risk factor assessment}

1. Alcohol intake: more than one drink per day - i.e. up to $24 \mathrm{gm}$ of pure alcohol per day

2. Smoking: $\geq 15$ pack years

3. NSAID'S: chronic use i.e. more than 3 times a week for more than 3 months.

\section{Exclusion criteria}

Age below 18 years, pregnant women, those who have not consented to participate in the study were not included.

Those with lower gastrointestinal bleeding - due to haemorrhoids, anal fissure, IBD, diverticulosis, CRC- were excluded.

\section{Results}

A total of 100 patients presenting with upper gastrointestinal bleeding were enrolled in this study. The history of patient's illness, any risk factors were noted. They were then evaluated for presence of peptic ulcer disease. Diagnosis of peptic ulcer was confirmed by endoscopy. The aim of the study is to know the burden of peptic ulcer disease in patients with upper gastrointestinal bleeding.

Table 1: Frequency distribution of study population according to their age. $(n=100)$

\begin{tabular}{|c|c|c|}
\hline S.no & Characteristics & No. of patients \\
\hline & Age & 9 \\
\hline 1 & $30-40$ years & 26 \\
\hline 2 & $40-50$ years & 33 \\
\hline 3 & $50-60$ years & 24 \\
\hline 4 & $60-70$ years & 5 \\
\hline 5 & $70-80$ years & 3 \\
\hline 6 & $80-90$ years & \\
\hline
\end{tabular}

The age distribution in the study population ranged from 30 years to 90 years. With the youngest being 33 years of age and oldest 86years. The mean age was 55 years.

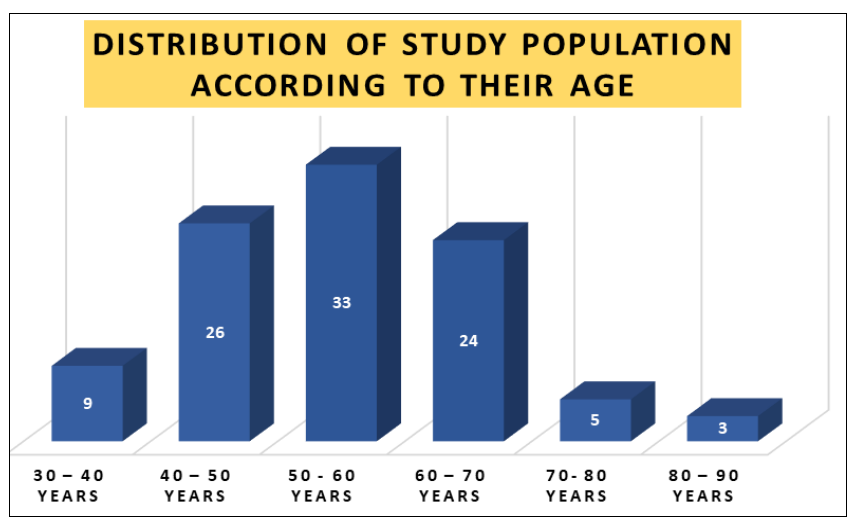

Fig 1: Distribution of study population according to their age

Table 2: Frequency distribution of study population according to their sex. $(n=100)$

\begin{tabular}{|c|c|c|}
\hline S.no & Characteristics & No. of patients \\
\hline & Sex & 76 \\
\hline 1 & Male & 24 \\
\hline 2 & Female & \\
\hline
\end{tabular}

Out of 100 cases 76 were males and 24 were females, giving a M: F ratio of 3:1 


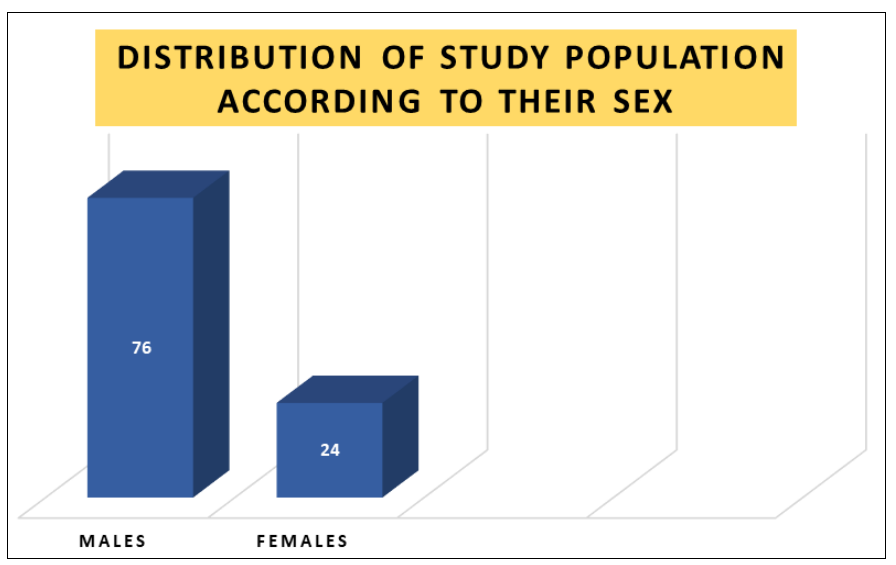

Fig 2: Distribution of study population according to their sex

Table 3: Frequency distribution of study population according to their risk factors $(n=100)$

\begin{tabular}{|c|c|c|c|}
\hline S.no & Characteristics & No. of patients & No. of patients \\
\hline & Risk factors & Yes & No \\
\hline 1 & Alcohol & 32 & 68 \\
\hline 2 & Smoking & 41 & 59 \\
\hline 3 & NSAID'S & 8 & 92 \\
\hline
\end{tabular}

Risk factors included alcohol intake which was seen in 32 patients, smoking was seen in 41, and NSAID's use was seen in 8 patients; out of a total of 100 patients

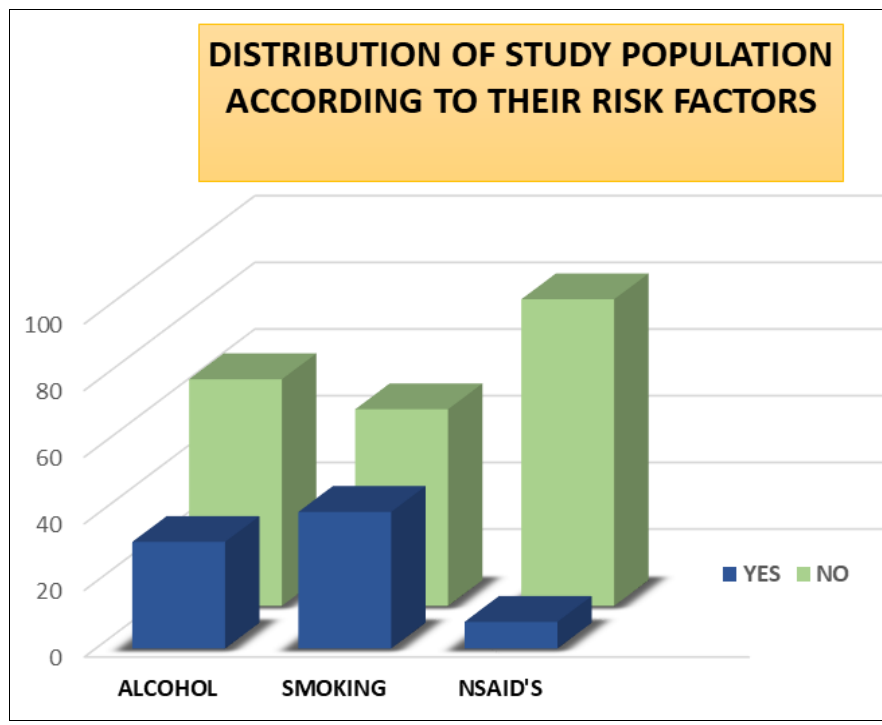

Fig 3: Distribution of study population according to their risk factors

Table 5: Frequency distribution of study population according to peptic ulcers $(n=100)$

\begin{tabular}{|c|c|c|c|}
\hline S. no & Characteristics & No. of patients & No. of patients \\
\hline & Peptic ulcer & Yes & No \\
\hline 1 & Gastric Ulcer & 21 & 76 \\
\hline 2 & Duodenal Ulcer & 24 & 79 \\
\hline 3 & Gastric and Duodenal Ulcer & 7 & 93 \\
\hline 4 & Esophageal Ulcer & 1 & 99 \\
\hline
\end{tabular}

In 100 patients presenting with upper gastrointestinal bleed, frequency of peptic ulcer disease was 53, of which 24 were duodenal ulcer, 21 were gastric ulcer,7 were both gastric and duodenal ulcer and 1 was an esophageal ulcer.

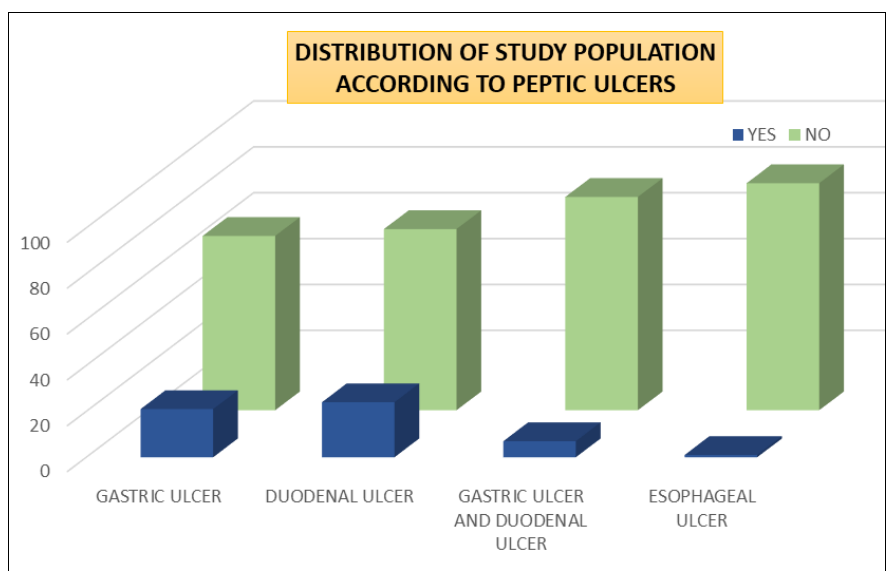

Fig 4: Distribution of study population according to Peptic Ulcers

\section{Discussion}

Peptic ulcer disease is common with a lifetime prevalence in the general population of $5-10 \%$ and an incidence of $0.1-0.3 \%$ per year ${ }^{[5]}$. Peptic ulcer is an erosion of gastro-duodenal mucosa due to detrimental effects of acid-peptic over-activity. This exposes underlying tissue to the digestive action of gastric and duodenal secretions. The ulcer bores through the muscularis and extending into submucosa or deeper. It usually involves the stomach and proximal part of the duodenum. It can also involve in the lower esophagus, distal part of duodenum, and jejunum. Peptic ulcer causation is almost always attributed to either $H$. pylori infection or chronic NSAID use. The pathogenesis of peptic ulcer disease may be considered as a combination scenario involving an imbalance between defensive factors (mucus-bicarbonate layer, prostaglandins, cellular regeneration, mucosal blood flow) and aggravating factors (hydrochloric acid, pepsin, ethanol, bile salts, drugs) ${ }^{[6]}$.

Both gastric and duodenal ulcer diseases are strongly associated with Helicobacter Pylori. H. pylori colonizes the gastric antrum first, causing non-atrophic antral gastritis; depletion of somatostatin producing delta cells. Thus, unchecked levels of gastrin lead to gastric acid hypersecretion. H. pylori also produces cytotoxins that inhibit duodenal production of bicarbonate, reducing mucosal protection. All of the above culminate to form a duodenal peptic ulcer. From the antrum, $H$. pylori gradually creeps on to the corpus. Here it causes patchy, multi-focal atrophic gastritis; loss of parietal and G-cells. This causes decrease in base line acid secretion, ultimately leading to decreased mucosal protection. This coupled with evasive action of urease to $\mathrm{HCl}$ and cytotoxins of $H$. pylori form a gastric peptic ulcer. Duodenal ulcers are four times more common than gastric ulcers ${ }^{[3]}$. Nonsteroidal anti-inflammatory drugs (NSAIDs) initiate gastroduodenal ulceration and promote complications such as bleeding and perforation ${ }^{[7]}$. Apart from being a direct mucosal irritant, NSAID's block COX pathways and prostaglandin production. Physiologically prostaglandins are mucosal protectants.

Alcohol consumption and cigarette smoking are two etiologic factors that have a close relationship with peptic ulcer diseases ${ }^{[8]}$. Smoking brings about numerous changes in gastric mucosa, with the end result being ulceration. These include reduction of epidermal growth factor, nitric oxide synthesis, blood flow to mucosa, and proliferation of cells. Smoking causes direct injury to tissue due to free radical production. Alcohol irritates the gastric mucosa and induces acidity. Alcohol also affects the mucosal barrier and histology. 
Common presentation of peptic ulcer disease includes epigastric pain, bloating, early abdominal fullness, nausea and vomiting, unintentional weight loss/weight gain. In the current study population presenting GI symptoms were pain abdomen, epigastric pain, heart burn, bloating, regurgitation, and early satiety. Clinical features may vary depending upon the location of the ulcer. Gastric and duodenal ulcers can be differentiated based on the timing of their symptoms in relation to meals. Gastric ulcer - pain aggravated by meals; duodenal ulcer - pain relieved by meals. Symptoms of dyspepsia are more often seen with gastric ulcers than duodenal ulcers. Nocturnal pain is a feature of duodenal ulcers.

When an ulcer remains undiagnosed or untreated it can wreak havoc by digging deep into the gut wall. Failure to abstain from risk factors such as alcohol, smoking, NSAIDs can also lead to complications. Potential complications of an ulcer are upper GI bleeding, perforation, gastric outlet obstruction, and malignant transformation (more common with gastric ulcers).

A proper history, focusing on symptoms pertaining to meal time, dietary intake and identification of risk factors is important to detect peptic ulcer disease. H. pylori can be detected by various methods - serology- Antibodies to H. pylori. Stool antigen test, rapid urine test, Urine-based ELISA, and urea breath test. Endoscopic biopsy is only recommended for refractory peptic ulcers and suspected antibiotic resistance. Diagnosis of peptic ulcer can be confirmed either invasively or non-invasively. Esophagogastroduodenoscopy (EGD) is the Gold standard and most accurate diagnostic test with sensitivity and specificity up to $90 \%$ in diagnosing gastric and duodenal ulcers ${ }^{[9]}$. Contrast CT -Barium swallow is done in cases where esophagogastroduodenoscopy is contraindicated. Contrast CT abdomen is useful in diagnosis of complications of peptic ulcer like perforation or gastric outlet obstruction. Serum gastrin levels should be checked in suspected case of Zollinger - Ellison syndrome.

Acute upper GI bleed can be a potentially life threatening abdominal emergency and is a frequent cause of hospitalization. Upper gastrointestinal bleed can be defined as bleeding from a source proximal to the ligament of Treitz. It can either be variceal or non-variceal bleeding. Variceal bleeding is a complication of end stage liver disease. Whereas, non-variceal bleeding is commonly associated with peptic ulcer disease. Peptic ulcers wear away the mucosa and cause GI bleed.

Peptic ulcer remains the most common cause of upper gastrointestinal bleeding. A recent review on the epidemiology of complicated peptic ulcer disease found that hemorrhage was by far the most common complication of peptic disease, with a reported annual incidence of hemorrhage in the general population ranging from 0.02 to $0.06 \%{ }^{[10]}$. Ulcers tend to bleed when they erode into a blood vessel. The bleed can be minimal, slow, long term bleed causing iron deficiency anemia. When medium- or large-sized arteries are eroded it can cause massive hemorrhage. An acute bleed can either present as hematemesis i.e. coffee ground emesis or melena i.e. black tarry stools. A brisk bleed can give an uncommon presentation of hematochezia.

In the present investigated study population, out of 100 cases of upper GI bleed, 53 were diagnosed with peptic ulcer disease, i.e. more than half of the cases. Bleeding from duodenal ulcers is four times more common than from gastric ulcers ${ }^{[11]}$. In our study we found that out of 53 cases of peptic ulcers 24 were duodenal ulcer, 21 were gastric ulcer, 7 were with both gastric and duodenal ulcer and 1 was an esophageal ulcer. Upper gastrointestinal bleeding is twice as common in men as in women and increases in prevalence with age $(>60 \mathrm{y})^{[12]}$. Out of 100 cases of upper GI bleed 76 were males and 24 were females, giving a M: F ratio of 3:1, the youngest being 33 years of age and oldest 86years. The mean age was 55 years.

\section{Conclusion}

Anti-secretory drugs like proton-pump inhibitors and $\mathrm{H} 2$ receptor antagonists are widely used to counteract the ill effects of acid hypersecretion in peptic ulcer disease. PPI's along with antibiotic coverage to eradicate $H$. pylori infection is the mainstay treatment for peptic ulcers. Despite introduction of triple/quadruple therapy for peptic ulcer disease, the morbidity and mortality caused by it is still cause for concern.

Peptic ulcers constitute the majority of upper GI bleeds, which left unmonitored, present as massive hemorrhage requiring intensive intervention. Thus, in a suspected or confirmed case of peptic ulcer, evaluation for any bleeding is a must. This can be done by testing haemoglobin and haematocrit levels, doing a complete blood picture along with platelet count. Stool should be tested for occult blood. Patients with peptic ulcer who require regular treatment with NSAID's (e.g. Rheumatoid arthritis) should be prescribed selective COX inhibitors or drugs that coat the ulcer (e.g. Sucralfate). Any patient who smokes or consumes alcohol should be counselled regarding the dangers of continuing the habit. Strict abstinence should be maintained to avoid further bleeding complications of the ulcer. Any patient above 65 years of age should be regularly monitored for any signs of bleeding due to the ulcer.

There has been a drastic reduction in the incidence and prevalence peptic ulcer disease, however timely detection and management of any upper GI bleed can reduce the burden of peptic ulcer morbidity.

\section{References}

1. Lin KJ, García Rodríguez LA, Hernández-Díaz S. Systematic review of peptic ulcer disease incidence rates: do studies without validation provide reliable estimates? Pharmacoepidemiol Drug Saf 2011;20:718.

2. Lanas A, Chan FKL. Peptic ulcer disease. Lancet 2017;390(10094):613-624.

3. Malik TF, Gnanapandithan K, Singh K. Peptic Ulcer Disease. [Updated 2021 Jul 29].

4. Byakodi KBSH, Teggimani V, Kabade N, Hiregoudar A, MRV. Factors affecting morbidity and mortality in peptic ulcer perforation. International Surgery Journal 2018;5(4);1335-1340.

5. Tarasconi A, Coccolini F, Biffl WL et al. Perforated and bleeding peptic ulcer: WSES guidelines. World J Emerg Surg 2020;15:3.

6. Prabhu V, Shivani A. An overview of history, pathogenesis and treatment of perforated peptic ulcer disease with evaluation of prognostic scoring in adults. Ann Med Health Sci Res 2014;4(1):22-29.

7. Scheiman JM. NSAID-induced peptic ulcer disease: a critical review of pathogenesis and management. Dig Dis 1994;12(4):210-22.

8. Ko JK, Cho $\mathrm{CH}$. Alcohol drinking and cigarette smoking: a "partner" for gastric ulceration. Zhonghua Yi XueZaZhi (Taipei) 2000.

9. ASGE Standards of Practice Committee. Banerjee S, Cash BD, Dominitz JA, Baron TH, Anderson MA, BenMenachem $\mathrm{T}$ et al. The role of endoscopy in the management of patients with peptic ulcer disease. Gastrointest Endosc 2010;71(4):663-8. 
10. Lau JY, Sung J, Hill C, Henderson C, Howden CW, Metz DC. Systematic review of the epidemiology of complicated peptic ulcer disease: incidence, recurrence, risk factors and mortality. Digestion 2011;84:102-13.

11. ACS/ASE Medical Student Core Curriculum Gastrointestinal Hemorrhage American College of Surgeons Division of Education (1-29-2018).

12. Bennie Ray Upchurch Upper Gastrointestinal Bleeding (UGIB) Updated: Sep 01, 2021.

https://emedicine.medscape.com/article/187857-overview. 